NASA Technical Memorandum 101574

\title{
AEROSERVOELASTIC MODELING AND APPLICATIONS USING MINIMUM-STATE APPROXIMATIONS OF THE UNSTEADY AERODYNAMICS
}

Sherwood H. Tiffany and Mordechay Karpel

\section{APRIL 1989}

(HASA-TH-101574) AEROSERVOEL ASTIC HODEL ING

N $89-24308$

AND APPL ICATIONS USING HINIHOH-STATE

APRBOXI HATIONS OF THE UASTEADY AERODINAH ICS

(Na SA. Langley Research Center) $13 \mathrm{p}$ Unclas

C $\stackrel{P}{S C L} \quad 01 \mathrm{C} \quad \mathrm{G} 3 / 05 \quad 0212638$

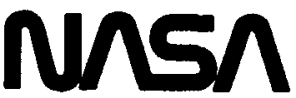

National Aeronautics and

Space Administration

Langley Research Center

Hampton, Virginia 23665-5225 


\title{
AEROSERVOELASTIC MODELING AND APPLICATIONS USING MINIMUM-STATE APPROXIMATIONS OF \\ THE UNSTEADY AERODYNAMICS
}

\author{
Sherwood H. Tiffany ${ }^{\dagger}$ \\ Aeroservoelasticity Branch \\ Structural Dynamics Division \\ NASA Langley Research Center \\ Hampton, Virginia 23665-5225 \\ and \\ Mordechay Karpel \\ Senior Research Associate \\ Department of Aeronautical Engineering \\ Technion - Israel Institute of Technology \\ Haifa, Israel
}

\begin{abstract}
Various control analysis, design, and simulation techniques for aeroelastic applications require the equations of motion to be cast in a linear time-invariant state-space form. Unsteady aerodynamics forces have to be approximated as rational functions of the Laplace variable in order to put them in this framework. For the Minimum-State method, the number of augmenting states representing the unsteady aerodynamics is a function only of the number of denominator roots in the rational approximation. Results are shown of applying various approximation enhancements (including optimization, frequency dependent weighting of the tabular data, and constraint selection) with the Minimum-State formulation to the Active Flexible Wing wind-tunnel model. The results demonstrate that good models can be developed which have an order of magnitude fewer augmenting aerodynamic equations than more traditional approaches. This reduction facilitates the design of lower order control systems, analysis of control system performance, and near real-time simulation of aeroservoelastic phenomena.
\end{abstract}

\section{Introduction}

The equations of motion of a flexible aircraft contain unsteady generalized aerodynamic force terms which are transcendental functions. The availability of efficient linear systems algorithms used in acroservoclastic analysis and design has provided strong motivation to approximate the unsteady acrodynamic forces as rational functions of the Laplace variable, refs. 1-5. Such rational function approximations (RFA's) allow the aeroservoelastic equations of motion to be cast in a linear time invariant (LTI) state-space form, albeit with increased size of the state vector due to the RFA's. This increase in the number of states due to the RFA's is referred to as the acrodynamic dimension. There is always a trade off between how well the RFA's approximate the aerodynamic forces and the desire to keep the acrodynamic dimension small. The RFA formulations in the literature (e.g., refs. 1 - 12) have varying capabilitics to perform such a trade-off.

Currently there are three basic formulations used in approximating unsteady generalized aerodynamic forces for arbitrary motion using rational functions:

1. Least-squares (LS) -- references 1 and 5

2. Modified matrix-Pade' (MMP) -- reference $2,3,6$, and 7

3. Minimum-State (MS) --8 and 12

Extensions to these approaches were developed (refs. 9 11) which included the capability to enforce selected equality constraints on the RFA's and of optimizing the denominator coefficients in the rational functions using nonlinear programming techniques. For the MinimumState method, the number of augmenting states required to represent the unsteady aerodynamics is a function only of the number of denominator roots in the rational approximation; there are no multiple aerodynamic roots as there are for the Least-Squares formulation. Methods to determine critical frequency ranges bašed upon physical properties and to determine weightings of the individual tabular errors to improve approximations in those critical regions have been explored in reference 12 .

The evolution of advanced fighters has required that the aerodynamics, control systems, and structures disciplines be integrated into a unified aeroservoelastic technology which must be evaluated by sophisticated analytical methods and validated through the testing of wind-tunnel models. The Active Flexible Wing (AFW) program was a joint Rockwell/Air Force/NASA program (ref. 13) that involved the design, construction, and testing of a scaled aeroelastic wind-tunnel model. A follow-on cooperative effort between NASA and Rockwell is currently underway to investigate and evaluate the analysis, design and test methodologies associated with multi-input/multi-output digital control laws. The purpose of this paper is to demonstrate the various Minimum-State aerodynamic modeling techniques by applying them to a current AFW wind tunnel model to

\footnotetext{
${ }^{\dagger}$ Member AIAA
} 
develop valid low order mathematical models for the control system design, evaluation and simulation tasks associated with the cooperative effort.

The following pages present a review of the techniques involved. Results are also shown which demonstrate that good models can be developed which introduce an order of magnitude fewer aerodynamic state equations than more traditional approaches.

\section{Unsteady Aerodynamic Approximations}

In order to express the equations of motion in first-order form, the unsteady generalized aerodynamic force (gaf) coefficients $\left(Q_{i j}\right)$ must be defined in the complex Laplace $s$-domain, or nondimensionalized Laplace $p$-domain, $p=(c / 2 u) s$; here $\mathrm{u}$ is the freestream velocity and $c$ is a reference length (typically the mean acrodynamic chord). Since the gaf coefficients are computed at specified values of reduced frequencies $k_{n}$, it is necessary to generate complex $p$-domain functions by approximating the tabular values, $Q_{i j}\left(i k_{n}\right)$, of the gars as closely as possible, and then employing the concept of analytic continuation in a region near the imaginary axis. Figure 1 depicts the approximating process for a single element $Q_{i j}$ of $Q$, where

\begin{tabular}{|c|c|}
\hline $\boldsymbol{k}$ & reduced frequency, $(c / 2 u) \omega$ \\
\hline$Q_{i j}\left(i k_{n}\right)$ & $\begin{array}{l}\text { reduced-frequency domain tabular data } \\
\text { (identified by the open circles) }\end{array}$ \\
\hline$\hat{Q}_{i j}(i k)$ & approximating curve, $Q_{i j}(p)$, for \\
\hline$\hat{Q}_{i j}\left(i k_{n}\right)$ & $\begin{array}{l}p=i k \text { (corresponding to the solid line) } \\
\text { points along the approximating curve at } \\
\text { reduced frequencies, } k_{n} \text {, corresponding to th } \\
\text { tabular data (solid dots) }\end{array}$ \\
\hline$\varepsilon_{i j}\left(i k_{n}\right)$ & $\begin{array}{l}\text { is the approximation error between two } \\
\text { corresponding points (denoted by an arrow } \\
\text { between the points). }\end{array}$ \\
\hline
\end{tabular}
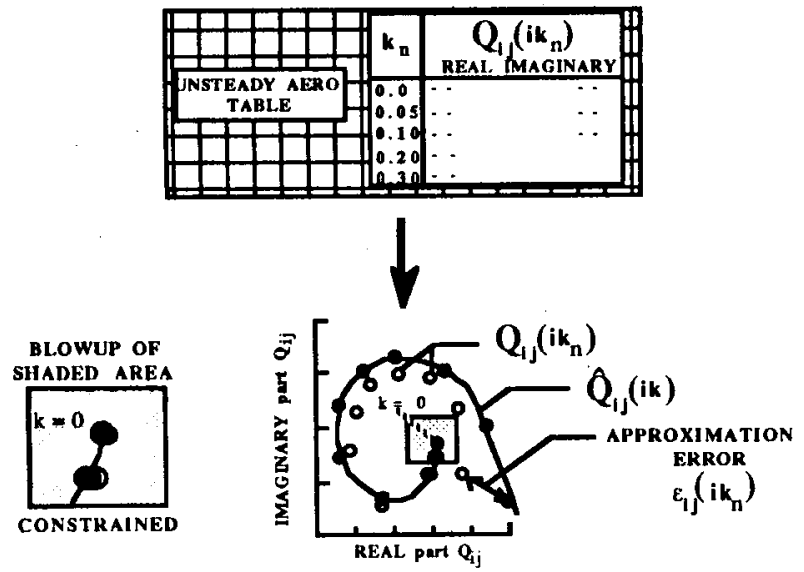

Figure 1.- Approximating aerodynamic tabular data for a single element of $Q$, employing constraints

The approximating function $\hat{Q}_{i j}(p)$ is determined in such a way as to minimize some least-square combina- tion of the errors, $\varepsilon_{i j}\left(i k_{n}\right)=\left|\hat{Q}_{i j}\left(i k_{n}\right)-Q_{i j}\left(i k_{n}\right)\right|$, between the approximating function at $p=i k_{n}$ and the tabular value $Q_{i j}\left(i k_{n}\right)$. It is possible that certain equality constraints or weighted constraints might be desirable to impose on some of the tabular values (such as the steady state $(k=0)$ point, shown in the shaded box).

\section{Review of Rational Function Approximations to Generalized Aerodynamic Forces}

The most common form of the approximating functions used currently for each generalized force coefficient, $Q_{i j}$, is a rational function of the nondimensional Laplace variable $p$. The normal form chosen is one in which the numerator polynomial is order 2 more than the denominator. This gives rise to the following partial fraction form for each element, $Q_{i j}$, where $n_{L}$ is the number of partial fractions and corresponds to the order of the overall denominator polynomial:

$$
\begin{array}{r}
\hat{Q}_{i j}(p)=\left(A_{0}\right)_{i j}+\left(A_{I}\right)_{i j} p+\left(A_{2}\right)_{i j} p^{2} \\
+\sum_{\ell=I}^{L}\left(A_{(\ell+2)}\right)_{i j} \frac{p}{p+b_{\ell}}
\end{array}
$$

which can be rewrituen in the Laplace domain as

$$
\begin{aligned}
\hat{Q}_{i j}(s)=\left(\bar{A}_{0}\right)_{i j} & +\left(\bar{A}_{l}\right)_{i j} s+\left(\bar{A}_{2}\right)_{i j} s^{2} \\
& +\sum_{\ell=l}^{n_{L}}\left(\bar{A}_{(\ell+2)}\right)_{i j} \frac{s}{s+\bar{b}_{\ell}}
\end{aligned}
$$

where

$$
\begin{aligned}
& \bar{b}_{\ell}=\frac{2 u}{c} b_{\ell} \\
& \bar{A}_{0}=A_{0} \\
& \bar{A}_{l}=\frac{c}{2 u} A_{l} \\
& \bar{A}_{2}=\left(\frac{c}{2 u}\right)^{2} A_{2} \\
& \bar{A}_{\ell+2}=A_{\ell+2}
\end{aligned}
$$

Because tabular data are determined for specified values of reduced frequency, $k_{n}$, the $Q_{i j}$ are actually defined only for these values of the nondimensionalized Laplace variable $p=i k_{n}$. It is desirable to use the above rational function form for the approximating functions in order to convert the transcendental equations of motion into linear, time-invariant, state-space form. The fact that the numerator is only order 2 more than the denominator implies the number of resulting equations associated with the acrodynamics is a function of the order of the denominator polynomials. The $A_{0}, A_{l}$, and $A_{2}$ terms can be included with the stiffness, damping, and mass terms, without any additional equations. 
When the matrix equations, which combine all the $\hat{Q}_{i j}$ 's, are formulated, the character of the partial fractions are expressed in various ways (ref. 11). The first,

$$
\begin{gathered}
\hat{Q}(p)=A_{0}+A_{l} p+A_{2} p^{2}+\hat{Q}_{L}, \text { where } \\
\hat{Q}_{L}=\sum_{\ell=l}^{n_{L}} A_{(\ell+2)} \frac{p}{p+b_{\ell}}
\end{gathered}
$$

is referred to simply as the "Least-Squares" (LS) formulation in which the number and values of denominator coefficients, $b_{\ell}$, are fixed for all $Q_{i j}$. The second,

$\hat{Q}_{j}(p)=\left\{A_{0}\right\}_{j}+\left\{A_{1}\right\}_{j} p+\left\{A_{2}\right\}_{j} p^{2}+\hat{Q}_{L j}$

where $\quad \hat{Q}_{L_{j}}=\sum_{\ell=l}^{n_{L_{j}}}\left\{A_{(\ell+2)}\right\}_{j} \frac{p}{p+b_{\ell_{j}}}$

for each column $j$, is a Modified Matrix-Pade' (MMP) formulation, which allows the number of denominator coefficients and their values to vary between columns. They are only fixed per column. The Minimum-State (MS) formulation,

$$
\begin{gathered}
\hat{Q}(p)=A_{0}+A_{1} p+A_{2} p^{2}+\hat{Q}_{L}, \text { where } \\
\hat{Q}_{L}=D(p l-R)^{-1} E p
\end{gathered}
$$

fixes the denominator coefficients as in the least squares, but additionally, the partial fraction numerator coefficients are determined as a coupled product of a premultiplying matrix, $D$, and a postmultiplying matrix, $E$. The diagonal matrix of aerodynamic roots is denoted by $R$; i.e.,

$$
R=\left[\begin{array}{lll}
\ddots & & \\
& -b_{\ell} & \\
& & \ddots
\end{array}\right], \quad \ell=1, \ldots, \mathrm{N}
$$

The resulting number of aerodynamic states introduced into the first order equations as a result of the MS formulation is $\mathrm{N}$. It is usually a little larger than $n_{L}$, but generally much smaller than either the aerodynamic dimension for the least-squares method, $n \xi^{\bullet} n_{L}$, or that of the modified matrix-Pade',

$$
\sum_{j=1}^{n_{\xi}} n_{L_{j}}
$$

The character of each $\hat{Q}_{L}$ and the acrodynamic dimensions for the different formulations is summarized in table 1.

\section{Constraints and Lag Coefficient Optimization}

Extended versions of the LS, MMP, and MS methods to fitting functions to aerodynamic tabular data are included in the ISAC program (ref. 14). The extensions include capabilities to select equality constraints and to perform a nonlinear optimization of the denominator coefficients (refs. 9-11). The equality constraints allow for more realistic modeling of the aerodynamics and for improved fits at critical points (such as flutter). Nonlinear optimization of the denominator coefficients allows improvement in the approximations without increasing their number by determining a better set of coefficients than might be chosen apriori.

\section{Table 1 RFA Matrix Formulations}

Aerodynamic Method

Least-Squares

Modified Matrix Pade

Minimum-State
Character of $\hat{Q}_{L}$

Common denominator coefficients in each $\hat{Q}_{i j}$

$$
\hat{Q}_{L_{i j}}=\sum_{\ell=l}^{n_{L}}(A(\ell+2))_{i j} \frac{p}{p+b_{\ell}}
$$

\section{Aerodynamic Dimension}

Different number of and values for denominator coefficients for each column, $Q_{j}$

$$
\hat{Q}_{L_{i j}}=\sum_{\ell=1}^{n_{L}}\left(A_{(\ell+2)}\right)_{i j} \frac{p}{p+b_{\ell_{j}}}
$$

$$
\sum_{j=1}^{n_{\xi}}{ }^{n} n_{L_{j}}
$$

Common denominator coefficients in each $\hat{Q}_{i j}$

$$
\hat{Q}_{L_{i j}}=\sum_{\ell=1}^{N} \frac{D_{i \ell} E_{\ell j} p}{p+b_{\ell}}
$$




\section{Minimum-State Equations of Motion}

The Minimum-State matrix equation for the unsteady aerodynamics is given by equation 4 . The resulting open-loop state-space equations of motion are:

$$
\begin{aligned}
& \left\{\begin{array}{l}
\dot{\xi} \\
\dot{\xi} \\
\dot{X}
\end{array}\right\}=\left[\begin{array}{ccccc}
0 & & \multicolumn{1}{c}{} & 0 \\
-\bar{M}_{\xi}^{-1} & \bar{K}_{\xi} & -M_{\xi}^{-1} & G_{\xi} & q \bar{M}_{\xi}^{-1} \\
0 & & E_{\xi} & \frac{2 u}{c} R
\end{array}\right]\left\{\begin{array}{l}
\xi \\
\dot{\xi} \\
X_{a}
\end{array}\right\}
\end{aligned}
$$

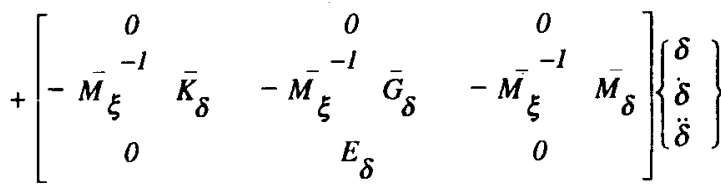

$$
\begin{aligned}
& +\left[\begin{array}{cccc}
0 & & 0 \\
-\bar{M}_{\xi}^{-1} & R_{g} & -\bar{M}_{\xi}^{-1} & G_{g} \\
I & & E_{g}
\end{array}\right]\left\{\begin{array}{c}
\alpha_{g} \\
\dot{\alpha}_{g}
\end{array}\right\}
\end{aligned}
$$

where $X_{a}=\left[s I-\frac{2 u}{c} R\right]^{-1}[E] s \xi$

The $\xi$ represents structural modes and the $\delta$ represents control modes. The submatrices are defined as follows:

$$
\begin{aligned}
& \bar{M}=M-q\left(\frac{c}{2 u}\right)^{2}\left[A_{2}\right] \\
& \bar{K}=K-q\left[A_{0}\right] \\
& K_{g}=-q\left\{A_{0}\right\}_{g} \\
& \bar{G}=G-q \frac{c}{2 u}\left[A_{1}\right] \\
& \bar{G}_{g}=-q \frac{c}{2 u}\left\{A_{l}\right\}_{g} \\
& \text { (mass matrix) } \\
& \text { (stiffness matrix) } \\
& \text { (damping matrix) } \\
& \alpha_{g}=\quad \text { nondimensional gust velocities } \\
& q \quad=\quad \text { dynamic pressure }
\end{aligned}
$$

$D, E$, and $R$ were defined in equations (4) and (5). The subscripts refer to column subsets involved; $\xi$ refers to vibration modes, $\delta$ refers to control modes, and $g$ to gust modes.

\section{Physical Weighting}

In ISAC (ref. 14) the inclusion of constraints is optional and those selected are imposed on a per column basis. A recently developed program, MIST (Minimum State), which has been interfaced with the ISAC program, provides the capability to include weights,

$$
w_{i j n}=\bar{w}_{i j n}\left(\max \left\{\frac{l}{\max _{i j}\left\{\tilde{w}_{i j}\right\}}, \frac{w_{c u t}}{\tilde{w}_{i j}}\right\}\right)
$$

where

$$
\tilde{w}_{i j}=\max _{n}\left\{\left.Q_{i j}\left(i k_{n}\right)\right|^{w_{i j n}}\right\} \text { and } 0 \leq w_{c u t} \leq 1
$$

on each $\varepsilon_{i j}\left(i k_{n}\right)$ to weight the importance of the fits at each tabular point $Q_{i j}\left(i k_{n}\right)$. Each $\bar{w}_{i j n}$ is a measure of importance which allows the fits to be improved at some points (at the possible expense of others) based upon physical properties without actually enforcing equality at the specified points (ref. 12) The method developed therein determines the measure of importance based upon partial derivatives of selected open-loop parameters with respect to the weighted term at a specified design point. For the vibration modes the weight of the fit at each value of $k$ is determined by the effective influence on the system determinant; for control modes, by the effect on Nyquist gains; for gust modes, by the effect on the response to continuous gusts; and for hinge moment terms, by the hinge moment response to control surface or gust excitations. The physical weights are $k$-dependent, but they are independent of normalization of the generalized acrodynamic force coefficients. The $w_{c u t}$ in equation (7) provides a means to specify the minimum of the maximum weighted magnitudes

$$
Q_{i j}^{\prime}=\max _{n}\left\{\left|Q_{i j}\left(i k_{n}\right)\right| w_{i j n}\right\}
$$

of each $Q_{i j}$. It can be shown that

$$
w_{c u t} \leq Q_{\ddot{y}}^{\prime} \leq 1 \text {. }
$$

Hence, a value of $w_{c u t}=0$ allows the full influence of physical weighting by setting the minimum $=0$; whercas $w_{c u t}=1$ forces the most sensitive point for each $Q_{i j}$ to be normalized or have a weighted magnitude of 1 .

The current MIST formulation requires the imposition of three equality constraints on each element $\hat{Q}_{i j}$. These equality constraints can be selected from various options, but must be the same for all $\hat{Q}_{i j}$. For the results shown herein, this requirement is not severe.

Data normalization is the basic measure of error used in ref. 9-11 which enforces fits equally (except for equality constraints) over a selected frequency range of tabular values in order to best meet the criterion for analytic continuation. However, since the concept of analytic continuation used to extend frequency domain functions, descibed by a finite set of tabular aerodynamic data, is approximate at best, physical weighting with optimization provides another tool for extending these functions in some reasonable fashion. Furthermore, varying the minimum of the maximum weighted magnitude, by varying the value of $w c u$ between 0 and 1 , provides a mechanism which allows the approximations to incorporate some combination of physical weighting and simple data normalization.

\section{AFW State-Space Modeling}

The AFW project requires various first-order models for different purposes such as low-order models for control law design and near real-time simulation and higher-order models for control system evaluation in batch simulation. 
For both types of simulation it was desired to generate a reliable mathematical model in which the mass matrix was constant, i.c. had no aerodynamic terms, in order to avoid repetitive matrix inversion. This implied that the $A_{2}$ coefficients in the rational approximations had to be zero for all rigid and clastic modes. To meet the low order requirements of design models and near real-time simulation, MS models were generated using 2 denominator coefficients. This resulted in an antisymmetric aeroservoelastic model with a total of 38 states. These states consisted of 22 rigid and clastic mode displacement and rate states, 12 actuator states, 2 gust states, and 2 aerodynamic states.

\section{Discussion of Results}

The following results are for the antisymmetric AFW model at Mach .9. There are 11 vibration modes (the roll mode is included in this group), 4 control modes and one gust. In each case, the aerodynamic approximations are constrained to match at $k=0$, the imaginary parts are are matched at $k=0.005$, and the $A_{2}=$ 0 . Furthermore, there is no constraint on the minimum that the maximum weighted magnitudes of $Q_{i j}$ must be (i.e., $w_{\text {cut }}=0.0$ ), corresponding to full inclusion of physical weighting.

Figure 2 is a set of three dimensional plots (from two different views) of the physically weighted magnitudes of each $Q_{i j}\left(i k_{n}\right)$ These weights were determined for a value of dynamic pressure $(q)$ near flutter. On each plot, $Q_{i j}$, $(i=1$ to 11$)$ are shown in a group for each $j$. For the vibration modes, $j$ increments from 1 to 11 , corresponding to the generalized coordinates. For the control modes, $j$ increments from 12 to 15 , corresponding to the leading edge inboard (LEI), trailing edge inboard (TEI), leading edge outboard (LEO), and the trailing edge outboard (TEO) control surfaces. There is one gust mode, corresponding to $j=16$. The peaks correspond to the tabular values of reduced frequency of highest influence on system characteristics. For example, for the roll mode (column 1) the system determinant is most sensitive to changes in the generalized acrodynamic force cocflicient near the third $k$ value, $k=0.01$, in the first row; i.c., the system is most sensitive to crrors in $Q_{11}\left(k_{3}\right)$.

Some of the rows have nearly zero weighted magnitudes for all values of $k$, which indicates that the system has minimal sensitivity to variation in these elements. The view on the left indicates that for the vibration modes, the diagonal terms, $Q_{i i}$, are the most sensitive. Referring to Table 2 for the values of in vacuum natural frequencies $\left(\omega_{v}\right)$, the view on the right indicates that the area of sensitivity is at $k$ values near $k_{v}$ of each mode. This can be seen more easily in figure 3 , which shows the weighted magnitudes of the most sensitive vibrational mode elements, $Q_{i i}$, at each tabular value of $k$. According to this plot, the system is most sensitive to errors in $Q_{66}$ at reduced frequencies between 0.6 and 0.8 . This plot also indicates that the generalized forces probably should have been calculated for additional valucs of reduced frequency between 0.6 and 1.5 , as the peaks are not clearly defined for some of the modes in this region. As indicated by figure 2 , the same denominator coefficients based on sensitivity for modes 1 through 5 should work for the control modes and gust in for this model. They have the same sensitivity range as the first 5 modes; i.e., they are not very sensitive to fits past $k=0.6$. In order to obtain a reliable model for all the modes selected however, it appears that the range of tabular values $(0.0<k<2.0)$ was appropriate for this configuration.

The determination of an optimal set of denominator coefficients for a Least-Squares fit is a fairly quick process (reference 11). For the Minimum-State approximation, however, nonlinear optimization of the denominator coefficients requires a three-fold, timeconsuming iteration process. In lieu of using nonlinear optimization, figure 3 was used to help select denominator coefficients for several cases. The selections, although not optimal, produced good fits, and results are presented in table 3 and figure 4 in order to show the reader the type of results which can be obtained with various selections of denominator coefficients.

\begin{tabular}{cccccc}
\hline \hline Table 2 & Frequency & parameters & $\left(\omega_{v}, \boldsymbol{k}_{v}\right)$ at $\mathbf{q}=\mathbf{0}$ and $\left(\omega_{f}, \boldsymbol{k}_{f}\right)$ at flutter \\
\hline \hline Mode & $\begin{array}{c}\omega_{\nu} \\
(\mathrm{rad} / \mathrm{sec})\end{array}$ & $k_{\nu}$ & $\begin{array}{c}\omega_{f}, \\
(\mathrm{rad} / \mathrm{sec})\end{array}$ & $k_{f}$ & $\begin{array}{c}\text { Most sensitive } \\
{\text { range } \mathrm{f}^{\dagger}}^{\dagger}\end{array}$ \\
\hline 1 & 2.39 & 0.009 & 9.34 & 0.034 & {$[0.0,0.1]$} \\
2 & 44.13 & 0.159 & 45.37 & 0.164 & {$[0.1,0.2]$} \\
3 & 49.37 & 0.178 & 53.93 & 0.195 & near 0.2 \\
$* 4$ & 82.23 & 0.297 & 73.05 & 0.263 & {$[0.2,0.3]$} \\
5 & 101.53 & 0.366 & 101.64 & 0.367 & near 0.4 \\
6 & 173.04 & 0.625 & 200.11 & 0.722 & {$[0.6,0.8]$} \\
7 & 240.65 & 0.869 & 217.93 & 0.787 & near 0.8 \\
8 & 248.93 & 0.899 & 248.79 & 0.898 & {$[0.8,1.0]$} \\
9 & 258.77 & 0.934 & 252.54 & 0.911 & {$[0.9,1.0]$} \\
10 & 313.74 & 1.132 & 313.95 & 1.133 & {$[1.0,1.2]$} \\
11 & 326.88 & 1.180 & 348.58 & 1.258 & near 1.2 \\
\hline \hline
\end{tabular}

*Flutter mode

$\dagger_{\text {range of }} \mathrm{k}$ for each mode in which system determinant is most sensitive to error 

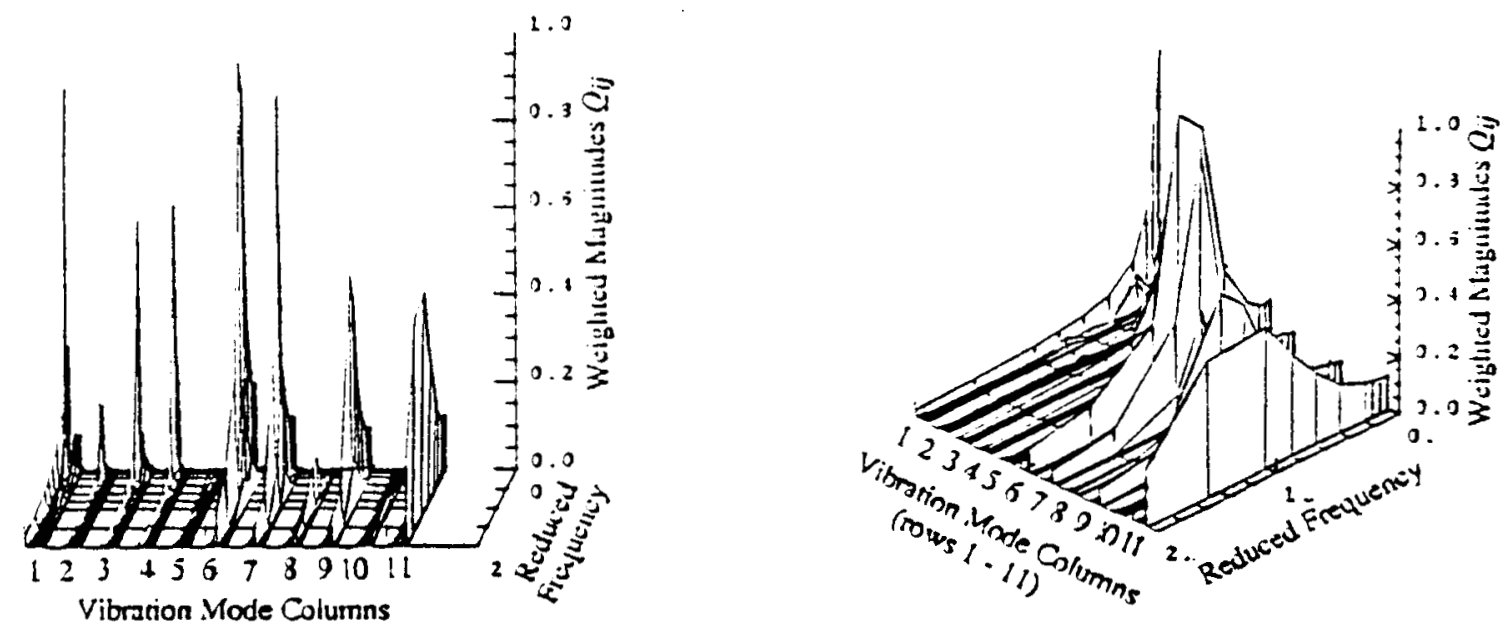

(rows l - il)

(a) Vibracion modes and rigid body roil
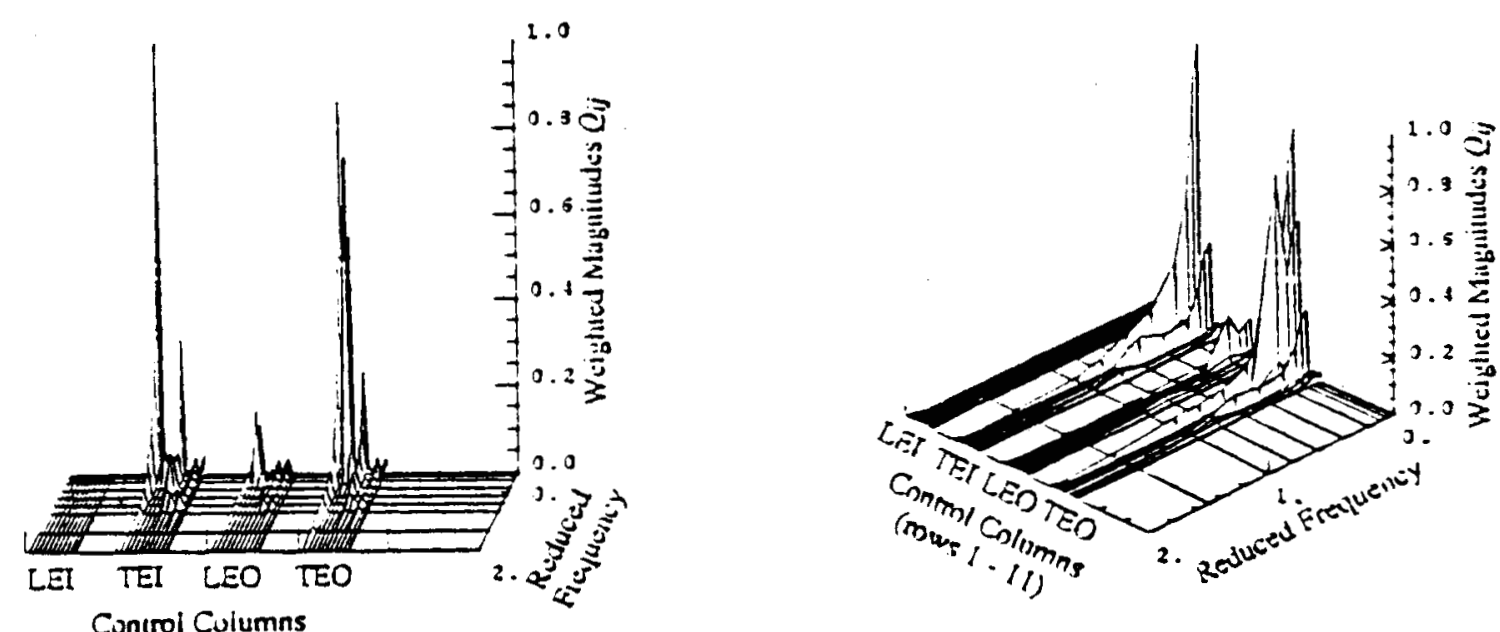

(b) Control modes
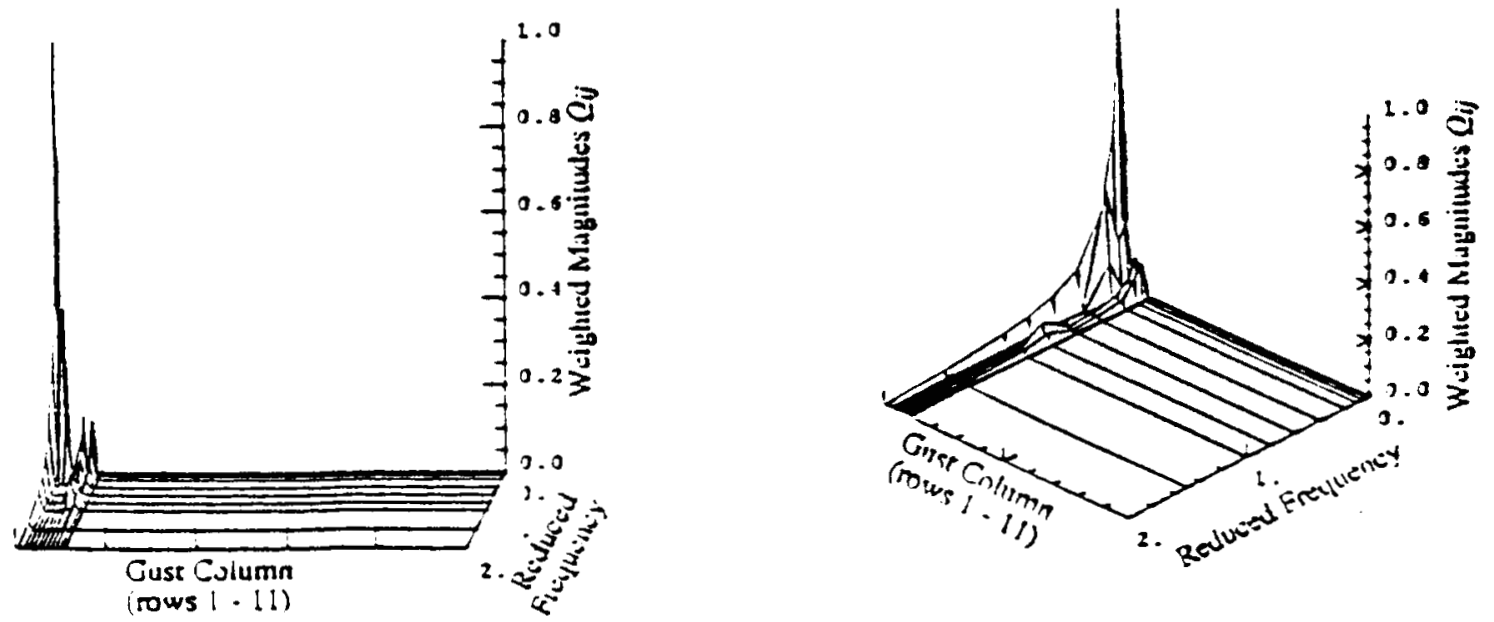

(c) Gust mode

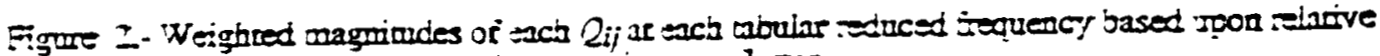
contrioution to system charscencics sent lluter 


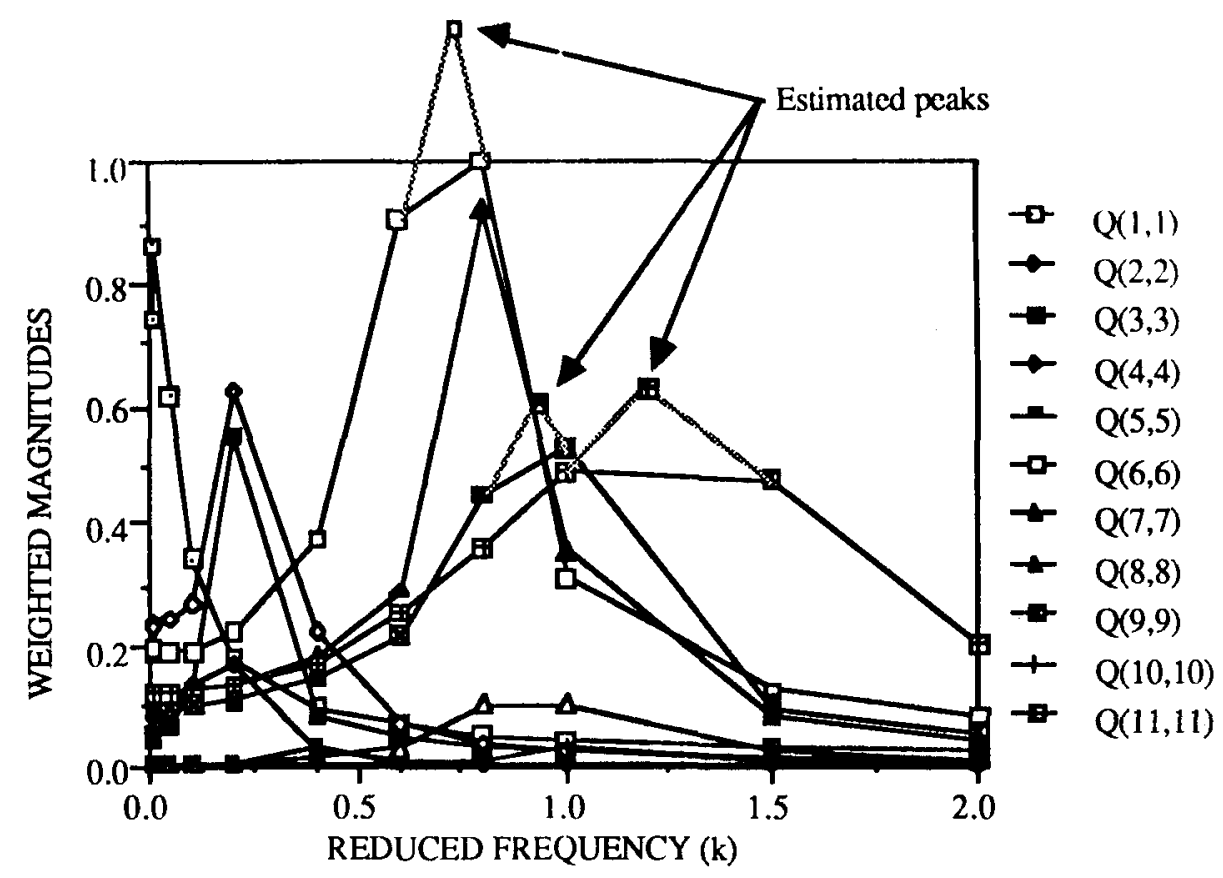

Figure 3.- Weighted magnitudes of most sensitive aerodynamic elements

Table 3 shows the choices made for the order of approximation equal to 1,2 , and 4 (equals the number of denominator coefficients). Also listed are the method for selection and the $\%$ crrors in flutter $q$ and frequency. The word 'unconstrained' refers to $k$ values for which the $\hat{Q}_{i j}$ are not constrained; i.e., those other than $k=0$ and $k=0.005$. The words 'estimated most sensitive $Q_{i j}$ ' refers to a case in which the estimated peaks (as identified in figure 3) are used to determine the most sensitive $Q_{i j}$. The $k$ 's corresponding to the $Q_{i j}$ with 'estimated peaks' are also estimated.
Figure 4 shows the open loop root loci of a baseline configuration and the best overall of the two and four-acrodynamic state cases as indicated in table 3 . The baseline used the tabular, frequency domain generalized aerodynamic forces and was generated using a $p-k$ determinant iteration process employing interpolated values (ref. 15). The other two were based on linear time invariant state-space methods. The Hassig form of implementation of the $p-k$ method (ref. 16) becomes less accurate as the approximation $Q(p)=Q(0+i k)$ degrades; hence the baseline result for the roll mode in figure 4 should not be regarded as accurate.

\begin{tabular}{|c|c|c|c|c|}
\hline $\begin{array}{c}\text { Order of } \\
\text { Approximation }\end{array}$ & $\begin{array}{c}\text { Values of Selected } \\
\text { Denominator coefficients }\end{array}$ & Method of Selection & $\begin{array}{c}q_{f} \\
\text { \%error }\end{array}$ & $\underset{\% \text { erro }}{\omega_{f}}$ \\
\hline \multirow[t]{4}{*}{1} & 0.8 & (unconstrained) $k$ of most sensitive $Q_{i i}$ & 0.06 & 0.27 \\
\hline & 1.0 & Mid-range $k$ & -0.07 & 0.29 \\
\hline & $0.722^{* \dagger}$ & $k_{f}$ of most sensitive $Q_{i j}$ & 0.13 & 0.26 \\
\hline & 0.625 & $k_{\nu}$ of most sensitive $Q_{i j}$ & 0.24 & 0.25 \\
\hline \multirow[t]{5}{*}{2} & $0.2,0.8^{*}$ & (unconstrained) $k$ 's of most sensitive $Q_{i j}$ 's & -0.07 & 0.32 \\
\hline & $0.625,0.869$ & $k_{v}$ 's of most sensitive $Q_{i j}$ 's & -0.87 & 0.38 \\
\hline & $0.297,0.625$ & (unconstrained) $k_{v}$ 's of most sensitive $Q_{i j}$ 's, for different $\mathrm{j}$ & 0.32 & 0.12 \\
\hline & $0.625,1.180$ & $k_{\nu}$ 's of estimated most sensitive $Q_{i j}$ 's & -0.78 & 0.37 \\
\hline & $0.4,1.5$ & Mid-range $k$ 's of least sensitive $Q_{i j}$ 's & -0.55 & 0.36 \\
\hline \multirow[t]{5}{*}{4} & $0.2,0.6,0.8,1.0$ & (unconstrained) $k$ 's of most sensitive $Q_{i j}$ 's & 0.64 & 0.00 \\
\hline & $0.2,0.8,1.0,1.5^{*}$ & (unconstrained) $k$ 's of most sensitive $Q_{i j}$ 's, for different $\mathrm{j}$ & 1.00 & 0.05 \\
\hline & $0.2,0.73,0.8,1.22$ & $k$ 's of estimated most sensitive (unconstrained) $Q_{i j}$ 's & 0.59 & 0.01 \\
\hline & $0.009,0.625,0.869,1.180$ & $k_{v}$ 's of estimated most sensitive $Q_{i j}$ 's & 0.09 & 0.12 \\
\hline & $0.159,0.366,0.899,1.132$ & $k_{\nu}$ 's of least sensitive $Q_{i j}$ 's & 0.98 & 0.05 \\
\hline
\end{tabular}

\footnotetext{
${ }^{*}$ Best frequency and damping properties at flutter for all modes for corresponding order of approximation

† Very little difference overall between this and other three cases
} 


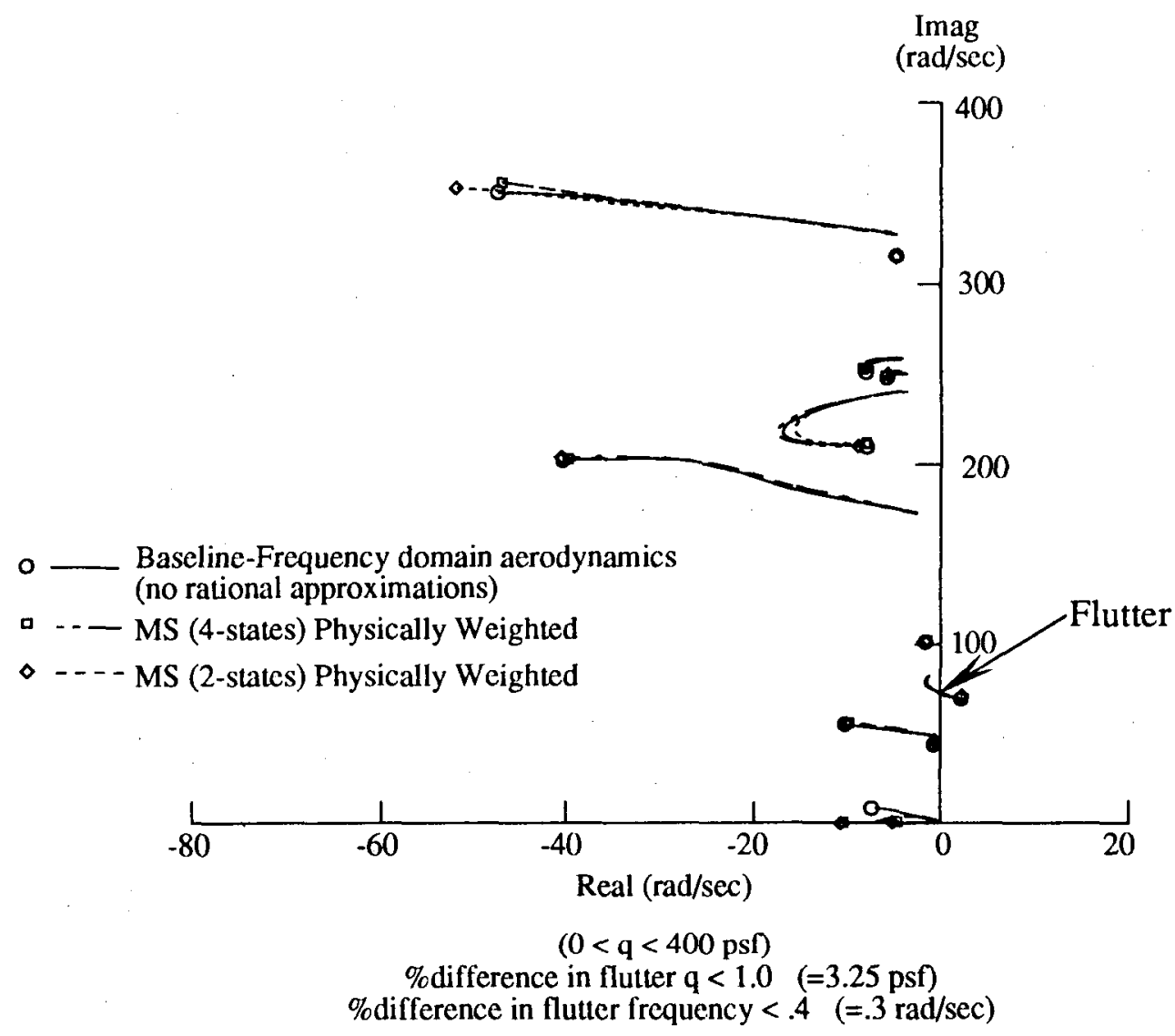

Figure 4.- Stability analysis root loci for the AFW antisymmetric modes at Mach 0.9.

\begin{tabular}{|c|c|c|c|c|c|c|}
\hline Order & $\begin{array}{c}\text { Values of Selected } \\
\text { Denominator Coefficients }\end{array}$ & Method of Selection & $\begin{array}{l}\text { sically Weighted } \\
\text { Total Error }\end{array}$ & $\begin{array}{l}\text { Normalized } \\
\text { Total Error }\end{array}$ & $\begin{array}{c}q_{f} \\
\text { \%error }\end{array}$ & $\begin{array}{c}\omega_{f} \\
\% \text { oerror }\end{array}$ \\
\hline 1 & 1.225 & Nonlinear Optimization & 0.32 & 17.75 & -0.18 & 0.31 \\
\hline 2 & $0.438,0.582$ & Nonlinear Optimization & 0.14 & 12.98 & -0.71 & 0.35 \\
\hline 3 & $0.431,0.917,1.314$ & Nonlinear Optimization & 0.07 & 19.22 & 0.58 & -0.01 \\
\hline 4 & $0.511,0.802,0.820,1.505$ & Nonlinear Optimization & 0.06 & 19.87 & 0.70 & 0.09 \\
\hline
\end{tabular}

In order to determine sets of aerodynamic approximations which consistently improve a chosen criterion as the order of the approximation increases (with increasing number of denominator coefficients), nonlinear optimization of the denominator coefficients must be employed. In this case the criterion for goodness of fit is the total physically weighted error. Table 4 shows the comparison of total physically weighted error $\left(w_{c u t}=0\right)$, as well as the total normalized error of refs. 9-11, and \% error in flutter $q$ and frequency for four optimized, physically wcighted MS approximations. Figure 5 shows the decreasing total physically weighted error criterion with increasing order. As would be expected since the sclection of denominator coeflicicents is based on optimum physically weighted crors, the normalized error is poor and does not improve with increasing order. There is no corresponding improvement in flutter $q$ and frequency, but figure 6 shows that there is an improving trend in overall frequency and damping characteristics at flutter of all the clastic modes with increasing order as compared to the frequency domain baseline flutter root characteristics.

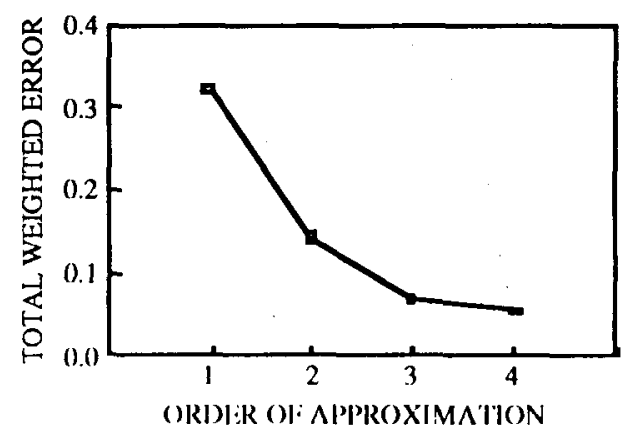

Figurc 5.- Comparison of total weighted errors for optimized physically weighted MS approximations 

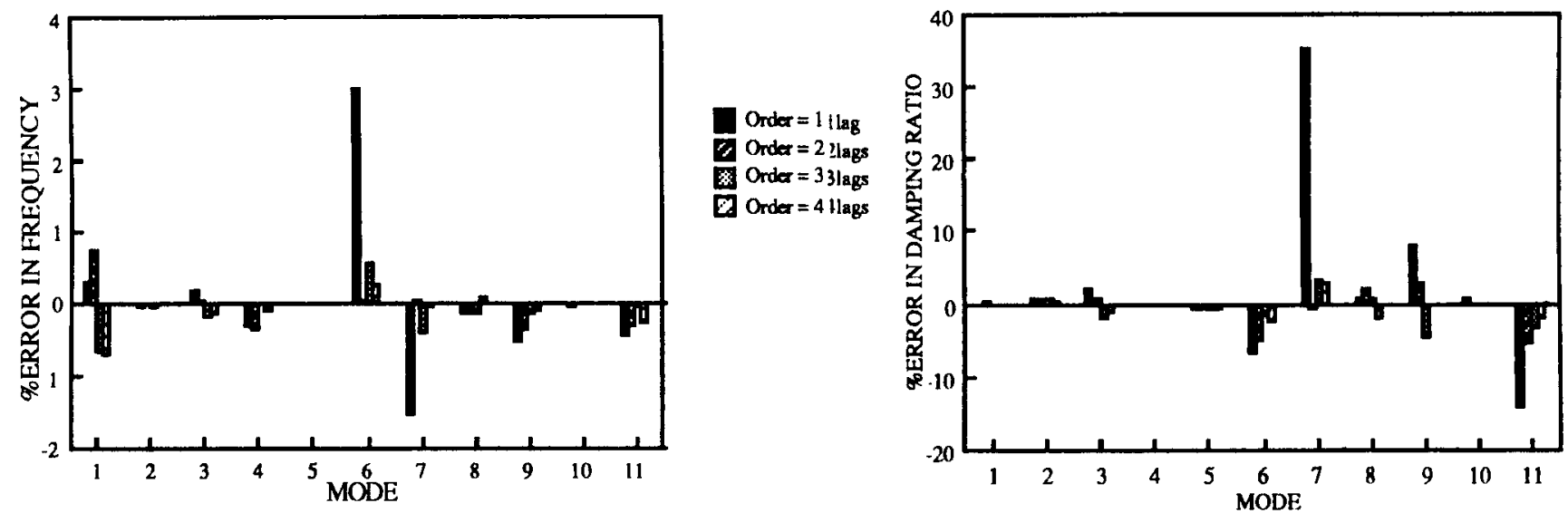

Figure 6.- Comparison of open loop flutter root characteristics for optimized physically weighted approximations

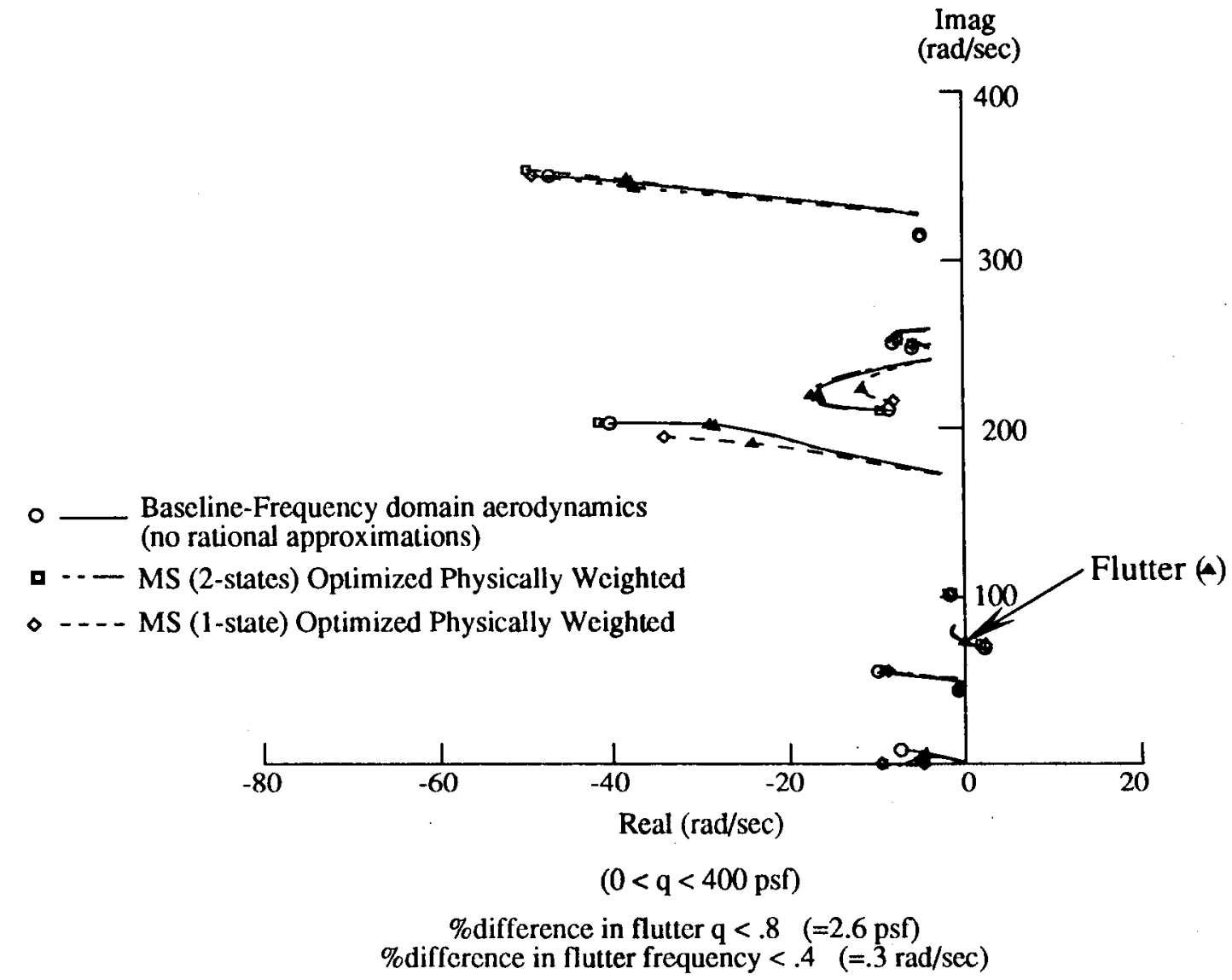

Figure 7.- Stability analysis root loci for the AFW antisymmetric modes at Mach 0.9 using optimized physically weighted Minimum-State approximations.

Figure 7 shows the stability analysis root loci of the 1-state and 2-state optimized, physically weighted MS approximations. The figure demonstrates that the optimized 1-state case matches the baseline case fairly well and that extremcly close correlation is achieved with the optimized 2-state approximation. The statements made with respect to the roll mode root locus in discussion of figure 4 apply here as well.
Figures 6 and 7 demonstrate that the physical weighting criterion for the vibrational modes is effective in achicving approximations which yicld accurate stability charactcristics for poles with low damping-ratios. It is interesting that agreement with the p-k root loci are also good for relatively large damping ratios despite the fact that the p-k approximation $Q(p)=Q(0+i k)$ is degrading; one only finds in the case of the roll mode where the $p-k$ 
approximation is clearly invalid that there is no consistent improvement in damping ratio as compared to the baseline with increasing order of approximation. Results (not shown) of Minimum-State approximations with the same equality constraints shown herein which reduce normalized error have higher \% errors in flutter $q$ and frequency and are much more sensitive to optimization of lag coefficients. Physical weighting tends to improve open-loop flutter characteristics and reduces the sensitvity to choices of acrodynamic roots. Corresponding control and gust response analyses, as well as closed loop flutter analyses would have to be presented in order to demonstrate the goodness of the physical weighting criteria for the control and gust modes.

\section{Concluding Remarks}

By combining various capabilities, namely the Minimum-State method, selectable constraints, optimal selection of denominator coefficients, as well as the determination and use of critical frequency ranges for approximating the tabular generalized aerodynamic forces with rational functions based upon physical properties, it is possible to obtain good, low order state-space mathematical models for design and simulation of aeroservoelastic systems. A state-space model with only two aerodynamic states predicted a flutter dynamic pressure with less than 0.8 percent error and showed good agreement in the root loci of all elastic modes as compared to conventional stability analysis. The significance of these results is that good models can be developed having an order of magnitude fewer augmenting aerodynamic equations than more traditional approaches. This reduction facilitates the design of lower order control systems, analysis of control system performance, and near real-time simulation of aeroservoelastic phenomena.

\section{References}

1. Sevart, Francis. D.: Development of Active Flutter Suppression Wind Tunnel Testing Technology. AFFDL TR-74-126.

2. Roger, Kenneth. L.: Airplane Math Modeling Methods for Active Control Design. Structural Aspects of Active Controls, AGARD CP-228, August 1977, pp. 4-1 - 4-11.

3. Vepa, Ranjan.: Finite State Modeling of Aeroelastic Systems, NASA CR 2770, 1977.

4. Edwards, John. W.: Unsteady Aerodynamic Modeling and Aeroelastic Control.. SUDAAR-504 (NASA Grant NGL-05-020-007), Dept. of Aeronautics and Astronautics, Stanford University, February 1977. (Available as NASA CR-148019.)

5. Abel, Irving.: An Analytical Technique for Predicting the Characteristics of a Flexible Wing Equipped with an Active Flutter-Suppression System and Comparison with Wind-Tunnel Data, NASA
TP-1367, 1979.

6. Dunn, Henry. J.: An Analytical Technique for Approximating Unsteady Aerodynamics in the Time Domain, NASA TP-1738, 1980.

7. Dunn, Henry. J.: An Assessment of Unsteady Acrodynamic Approximations for Time Domain Analysis. Proceedings of the Aeroservoelasticity Specialist Meeting, AFWAL-TR-84-3105, Vol. 1, U. S. Air Force, October 1984, pp. 98-115.

8. Karpel, Mordechay.: Design for Active and Passive Flutler Suppression and Gust Alleviation, NASA CR3482, 1981.

9. Tiffany, Sherwood. H.; and Adams, William M., Jr.: Fitting Aerodynamic Forces in the Laplace Domain: An Application of a Nonlinear Nongradient Technique to Multilevel Constrained Optimization, NASA TM-86317, 1984.

10. Tiffany, Sherwood. H.; and Adams, William. M., Jr.: Nonlinear Programming Extensions to Rational Function Approximations of Unsteady Aerodynamics. AIAA Paper 87-0854-CP presented at the AIAA/ASME/ASCE/AHS 28th Structures, Structural Dynamics, and Materials Conference, Monterey, California, April 1987.

11. Tiffany, Sherwood. H.; and Adams, William. M., Jr.: Nonlinear Programming Extensions to Rational Function Approximation of Unsteady Aerodynamic Forces. NASA TP-2776, 1988.

12. Karpel, Mordechay: Time-Domain Aeroservoelastic Modeling Using Weighted Unsteady Aerodynamic Forces. Submitted for publication in J. Guidance, Control, and Dynamics, Log No. G2460, March 1988.

13. Perry, Boyd III; Dunn, Henry J.; and Sanford, Maynard C.: Control Law Parameterization for an Aeroelastic Wind-Tunnel Model Equipped with an Active Roll Control System and Comparison with Experiment . NASA TM-100593, 1988.

14. Pecle, Ellwood. L.; and Adams, William. M., Jr.: $A$ Digital Program for Calculating the Interaction Between Flexible Structures, Unsteady Aerodynamics, and Active Controls. NASA TM80040, January 1979.

15. Adams, William M., Jr.; Tiffany, Sherwood H.; Newsom, Jerry R.; and Peele, Ellwood L.: STABCAR : A Program for Finding Characteristic Roots of Systems Having Transcendental Stability Matrices. NASA TP2165, 1984.

16. Hassig, Hermann J.: An Approximate True Damping Solution of the Flutter Equation by Determinant Iteration. J. Aircraft, Vol. 8, No. 11, Nov. 1971, pp. 885-889. 


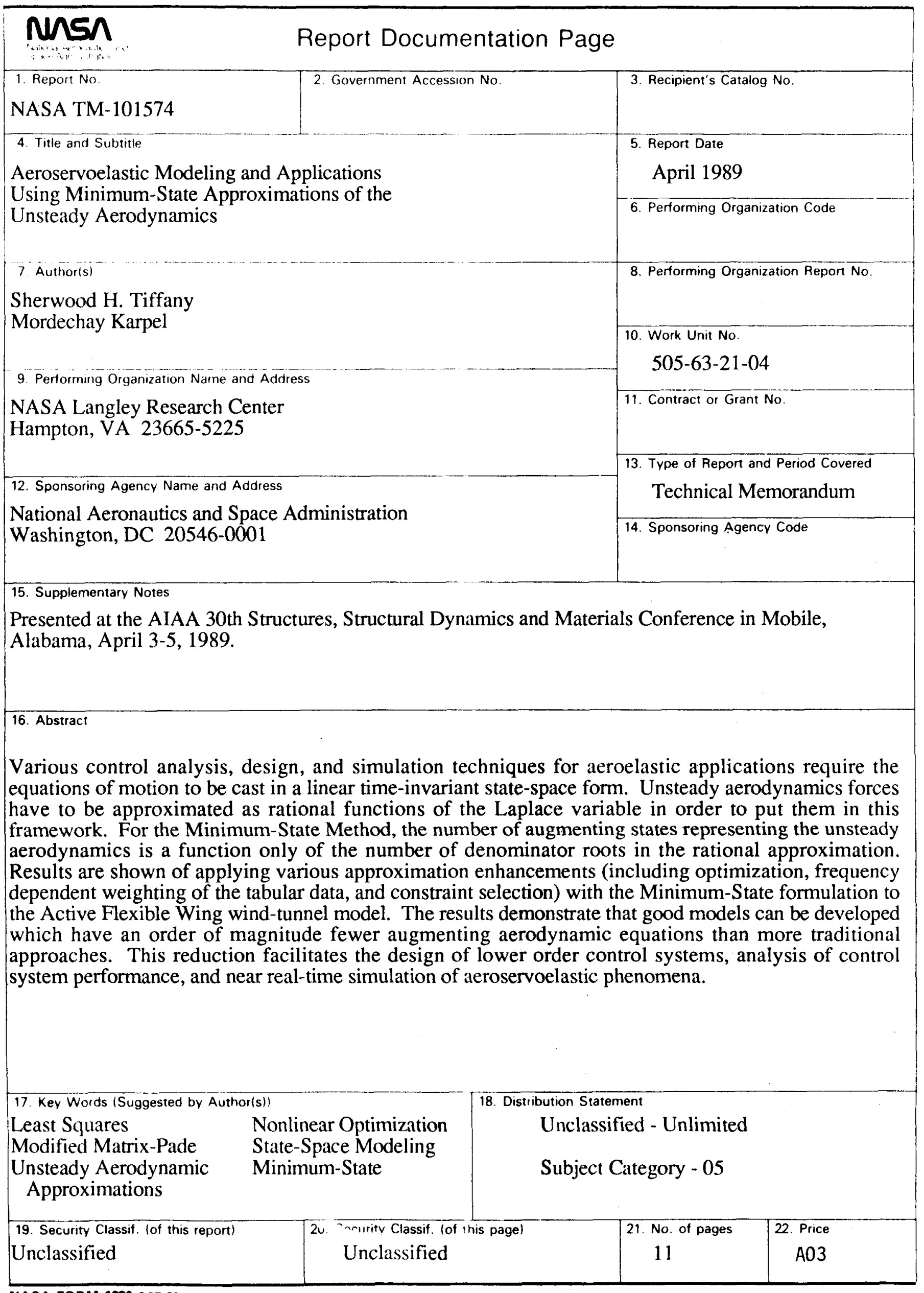

